\title{
The Use of Virtual Observatory Databases in Binary Star Research
}

\author{
Geraldine J. Peters \\ Space Sciences Center/Department of Physics \& Astronomy, University of Southern \\ California, Los Angeles, CA 90089-1341, USA \\ email: gjpeters@mucen.usc.edu
}

\begin{abstract}
The rapidly-accumulating archives of ground-based and spacecraft data worldwide that are being linked together through the International Virtual Observatory Alliance (IVOA) provide the binary star community with unparalleled opportunities for research. The main databases that are available to the astronomical community through the IVOA are discussed. Data from long-lasting spacecraft missions such as $I U E$ are especially valuable for studying long-term variability. Some examples of current research on close binary stars that is being carried through with UV spectra from the $I U E$ archive are presented. Included are the search for O-subdwarf companions to bright Be stars and some results from an ongoing investigation of the Double Periodic Variable phenomenon in Algol binaries.
\end{abstract}

Keywords. astronomical data bases: miscellaneous, (stars:) binaries: close, stars: emission-line, Be, ultraviolet: stars

\section{Introduction}

A vast amount of astronomical data of high quality from both spacecraft and groundbased facilities resides in public archives that are accessible to researchers worldwide. Spectroscopic and photometric data that span the electromagnetic spectrum from the gamma ray region to the infrared are available. UV and optical polarimetry also exists. These archived observations are now being linked together through the International Virtual Observatory Alliance (IVOA), an umbrella organization currently with members from 19 international scientific centers. As a result multiwavelength studies of astronomical objects are now commonplace. Especially valuable are data from long-lasting spacecraft missions such as IUE that generated a uniform set of FUV \& NUV spectra over a period of nearly two decades. The discovery of long-term variability cycles and studies of active binary stars with long periods are now possible. The availability of numerous advanced codes for the interpretation of the data, such as the ones discussed at this meeting, has enabled the astronomical community to discover and study phenomena that were unknown when the spacecraft mission was in operation. The new codes also allow researchers to determine fundamental parameters for stellar atmospheres, winds, disks, and other circumstellar material that represent an immense improvement over the earlier values.

In this paper the developing International Virtual Observatory Alliance and some major databases that are available through it are reviewed. Particularly useful for studying long-term activity in close binary stars are the archives of UV spectra from long-lived spacecraft missions such as $I U E$. Examples of a discovery that could never have been possible without the latter data and current research on the recently-identified Double Periodic variable (DPV)phenomenon in Algol binaries are presented. 


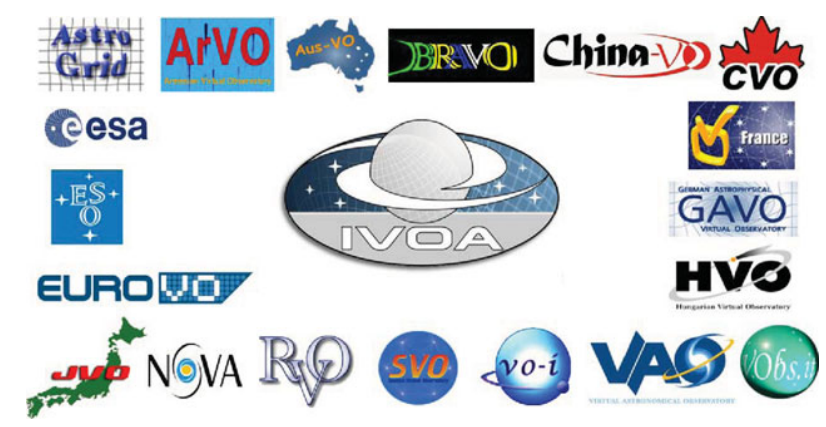

Figure 1. The logos for the IVOA and its member organizations.

\section{The International Virtual Observatory Alliance (IVOA)}

The International Virtual Observatory Alliance (IVOA, http://www.ivoa.net/) formed in 2002 is an umbrella organization currently with 19 members whose purpose is to combine archival data from spacecraft missions and ground-based observatories into one major site than can be accessed worldwide for astronomical research. Logos for the member institutions, which include programs from Argentina, Armenia, Australia, Brazil, Canada, China, Europe, France, Germany, Hungary, India, Italy, Japan, Russia, Spain, the United Kingdom, and the United States and inter-governmental organizations (ESA and ESO), are shown in Fig. 1. Working groups are establishing standards for the data formats and common software that will ultimately be approved by Commission 5 (Astronomical Data) of the International Astronomical Union.

The National Virtual Observatory of the United States (NVO, http://www.us-vo.org/) hosts an evolving website that allows researchers to find and retrieve astronomical data from international archives and data centers. Included are data from spacecraft, optical facilities, and catalogs such as those accessible through the VizieR $\dagger$. The Virtual Astronomical Observatory (VAO, http://www.usvao.org/ ) of the United States is developing software tools that will make efficient use of the information gathered through the NVO. Of note are the NVO links to spacecraft data currently in the Multimission Archive at STScI (MAST, http://archive.stsci.edu/), the High Energy Astrophysics Science Archive Research Center (HEASARC, http://heasarc.gsfc.nasa.gov/), and the Infrared Science Archive (IRSA, http://irsa.ipac.caltech.edu/). From the MAST website a researcher can link to the databases from the spacecraft missions listed in Table 1. High resolution UV spectra from Copernicus to the Far Ultraviolet Spectroscopic Explorer can be accessed through MAST as well as UV, optical, and infrared images from the Hubble Space Telescope, the Digitized Sky Survey, and the high precision optical photometry currently being delivered from the Kepler spacecraft. NASA's HEASARC is the source of spacecraft data on high energy phenomena. Links can be found to missions that produced gamma-ray to X-ray spectra and fluxes (cf. Table 2). HEASARC's databases also include FUV/NUV images and grism spectra from the Galaxy Evolution Explorer (GALEX) mission and microwave data from the Cosmic Background Explorer $(C O B E)$ and the Wilkinson Microwave Anisotropy Probe (WMAP). Databases in the IR and microwave regions are accessed through the NASA/IPAC Infrared Science Archive. Currently sixteen missions/projects are represented including data from the Infrared Astronomical Satellite (IRAS), Infrared Space Observatory (ISO), Spitzer Space Telescope,

$\dagger$ A joint effort of CDS (Centre de Données Astronomiques de Strasbourg and ESA-ESRIN (Information Systems Division). 
Table 1. Datasets Available Through the MAST

\begin{tabular}{|c|c|c|}
\hline Mission & Name & Type of Data \\
\hline \begin{tabular}{c|} 
ASTRO/HUT \\
ASTRO/UIT \\
ASTRO/WUPPE
\end{tabular} & $\begin{array}{c}\text { Hopkins Ultraviolet Telescope } \\
\text { Ultraviolet Imaging Telescope } \\
\text { Wisconsin Ultraviolet Photo-Polarimeter Experiment }\end{array}$ & $\begin{array}{l}\text { FUV spectra } \\
\text { FUV images } \\
\text { FUV polarimetry }\end{array}$ \\
\hline DSS & Digitized Sky Survey & Optical/NIR images \\
\hline EUVE & Extreme Ultraviolet Explorer & | EUV spectra \\
\hline FUSE & Far Ultraviolet Spectroscopic Explorer & |FUV spectra \\
\hline GALEX & Galaxy Evolution Explorer & $\begin{array}{l}\text { FUV/NUV images \& } \\
\text { LORES spectra }\end{array}$ \\
\hline GSC & Guide Star Catalog & Optical positions of stars \\
\hline HPOL & University of Wisconsin Polarimeter & $\mid \begin{array}{ll}\text { Optical } & \text { polarimetry, } \\
\text { photometry, and spectra }\end{array}$ \\
\hline $\mathrm{HST}$ & Hubble Space Telescope & $\begin{array}{l}\text { FUV/NUV, Optical, IR } \\
\text { spectra, photometry, \& } \\
\text { images }\end{array}$ \\
\hline IUE & International Ultraviolet Explorer & $\begin{array}{l}\text { HIRES/LORES FUV \& } \\
\text { NUV spectra }\end{array}$ \\
\hline Kepler & Kepler spacecraft & $\begin{array}{l}\text { High precision optical } \\
\text { photometry }\end{array}$ \\
\hline $\mathrm{OAO}-3$ & Copernicus satellite & | $\begin{array}{l}\text { HIRES FUV/NUV spec- } \\
\text { tra }\end{array}$ \\
\hline $\begin{array}{l}\text { ORFEUS-SPAS } \\
\text { ORFEUS/BEFS } \\
\text { ORFEUS/IMAPS } \\
\text { ORFEUS/TUES }\end{array}$ & $\begin{array}{c}\text { Orbiting and Retrievable Far and Extreme UV Spectrograph } \\
\text { Berkeley Extreme and Far-UV Spectrometer } \\
\text { Interstellar Medium Absorption Profile Spectrograph } \\
\text { Tübingen Echelle Spectrograph }\end{array}$ & $\begin{array}{l}\text { See below } \\
\text { HIRES FUV spectra } \\
\text { Very HIRES FUV spec- } \\
\text { tra HIRES FUV spectra }\end{array}$ \\
\hline VLA-FIRST & Very Large Array & $\begin{array}{l}\text { Faint images of sky at } \\
21 \mathrm{~cm}\end{array}$ \\
\hline XMM-OM & X-ray Multi-Mirror Telescope Optical Monitor & | LORES X-ray spectra \\
\hline
\end{tabular}

Wide-field Infrared Survey Explorer (WISE), and the Planck mission to study the cosmic microwave background.

The American Association of Variable Star Observers (AAVSO, http://www.aavso. org/) has been collecting and analyzing ground-based photometry from astronomers worldwide now for over 100 years. Their archive contains more than 20.5 million observations and they have established strong collaborations between professional and amateur astronomers. The All Sky Automated Survey (ASAS, http://www.astrouw.edu.pl/asas/) is a current photometric project in which more than $10^{7}$ stars are being monitored in the Johnson V and I bands from two small telescopes in Hawaii and Chile. Notable are the OGLE (Optical Gravitational Lensing Experiment, http://ogle.astrouw.edu.pl/) and MACHO (search for MAssive Compact Halo Objects, http://wwwmacho.anu.edu.au/) microlensing projects that have produced $>10^{9}$ optical observations of stars in the Magellanic Clouds and Galactic Bulge in the past two decades. Other databases include the RAVE (RAdial Velocity Experiment, http://www.rave-survey.aip.de/rave/) which is giving positions, distances and proper motions of more than $10^{6}$ stars, the ELODIE archive (http://atlas.obs-hp.fr/elodie/) of high resolution stellar spectra, and the Be Star Spectra (BeSS) database http://basebe.obspm.fr/basebe/) which contains 54,000 spectra of more than 600 different Be stars.

Perhaps the most valuable databases for investigating short and long-term activity in the close binaries are those from the International Ultraviolet Explorer (IUE) and Far 
Table 2. Datasets Available Through NASA's HEASARC

\begin{tabular}{|c|c|c|}
\hline Mission & Name/Description & | Type of Data \\
\hline AGILE & Italian Space Agency (ASI) spacecraft & $\begin{array}{l}\text { Gamma-ray/Hard X-ray detec- } \\
\text { tors }\end{array}$ \\
\hline $\mathrm{ASCA}$ & ASTRO-D, Japanese X-ray satellite & $\begin{array}{l}\text { X-ray photometry \& LORES } \\
\text { spectroscopy }\end{array}$ \\
\hline BeppoSAX & ASI/NIVR (Netherlands)/ESA X-ray satellite & | Soft/Hard X-ray detectors \\
\hline COBE & Cosmic Background Explorer & $\begin{array}{l}\text { Microwave/ FIR photometry \& } \\
\text { LORES spectroscopy }\end{array}$ \\
\hline CGRO & Compton Gamma Ray Observatory & | Gamma-ray photometry \\
\hline Chandra & Chandra X-ray Observatory & $\begin{array}{l}\text { HIRES X-ray imaging/photom- } \\
\text { etry \& spectroscopy }\end{array}$ \\
\hline EUVE & Extreme Ultraviolet Explorer & |EUV spectra \\
\hline Fermi & Fermi Gamma-ray Space Telescope & | Gamma-ray photometry \\
\hline GALEX & Galaxy Evolution Explorer & \begin{tabular}{|l} 
FUV/NUV images \& LORES \\
spectra
\end{tabular} \\
\hline HETE-2 & The High Energy Transient Explorer Mission & $\begin{array}{l}\text { Gamma-ray/X-ray burst pho- } \\
\text { tometry }\end{array}$ \\
\hline INTEGRAL & The INTErnational Gamma-Ray Astrophysics Laboratory & $\begin{array}{l}\text { Gamma-ray imaging/spectros- } \\
\text { copy }\end{array}$ \\
\hline ROSAT & The Róntgen Satellite & |X-ray imaging/photometry \\
\hline RXTE & The Rossi X-ray Timing Explorer Mission & | Rapid X-ray photometry \\
\hline Suzaku & The Suzaku Mission (ASTRO-E) & $\begin{array}{l}\text { X-ray imaging spectrometer \& } \\
\text { hard X-ray detector }\end{array}$ \\
\hline Swift & The Swift Gamma-Ray Burst Mission & $\begin{array}{l}\text { Gamma-ray burst detector, } \mathrm{X}- \\
\text { ray \& optical region telescopes }\end{array}$ \\
\hline WMAP & Wilkinson Microwave Anisotropy Probe & | Microwave imaging \\
\hline |XMM-Newton & ESA X-ray mission & | HIRES X-ray images \& spectra \\
\hline
\end{tabular}

Ultraviolet Spectroscopic Explorer (FUSE). Both produced high resolution, well-calibrated UV spectra over a long duration. IUE was launched on January 26, 1978 and operated for 18.75 years. This small Ritchey-Chretien telescope with an aperture of $45 \mathrm{~cm}$ was placed in an elliptical geosynchronous orbit with a perigee/apogee of $26000 / 46000 \mathrm{~km}$ that allowed it to be operated in real time by NASA and ESA with the observer present at either operations center. The IUE spacecraft yielded FUV (1150-1950 A, SWP) and NUV (1900-3200 $\AA$, LWR/LWP) spectra at resolutions of 0.1-0.3 $\AA$ (HIRES) and 6$8 \AA$ (LORES). Over 120,000 images were produced of which 2374 are of binary stars, including 556 SWP HIRES spectra. The FUSE spacecraft, launched on June 24, 1999, was operated for NASA and the French and Canadian Space Agencies for 8.3 years. It produced FUV spectra (950-1188 $\AA$ ) with a resolution of $0.05 \AA$. Over 6000 observations were made of which 195 were of binary stars (mostly from two large survey programs).

\section{Some Uses of Virtual Databases in Binary Star Research}

\subsection{Detection of $O$-Type subdwarfs}

The $I U E$ archive has proven to be especially valuable for detecting the presence of close sdO companions to bright Be stars. More than twenty years ago researchers were beginning to suspect that some Be stars may have been spun up to their very rapid rotation rates through the process of mass transfer. It seemed impossible to confirm the presence 

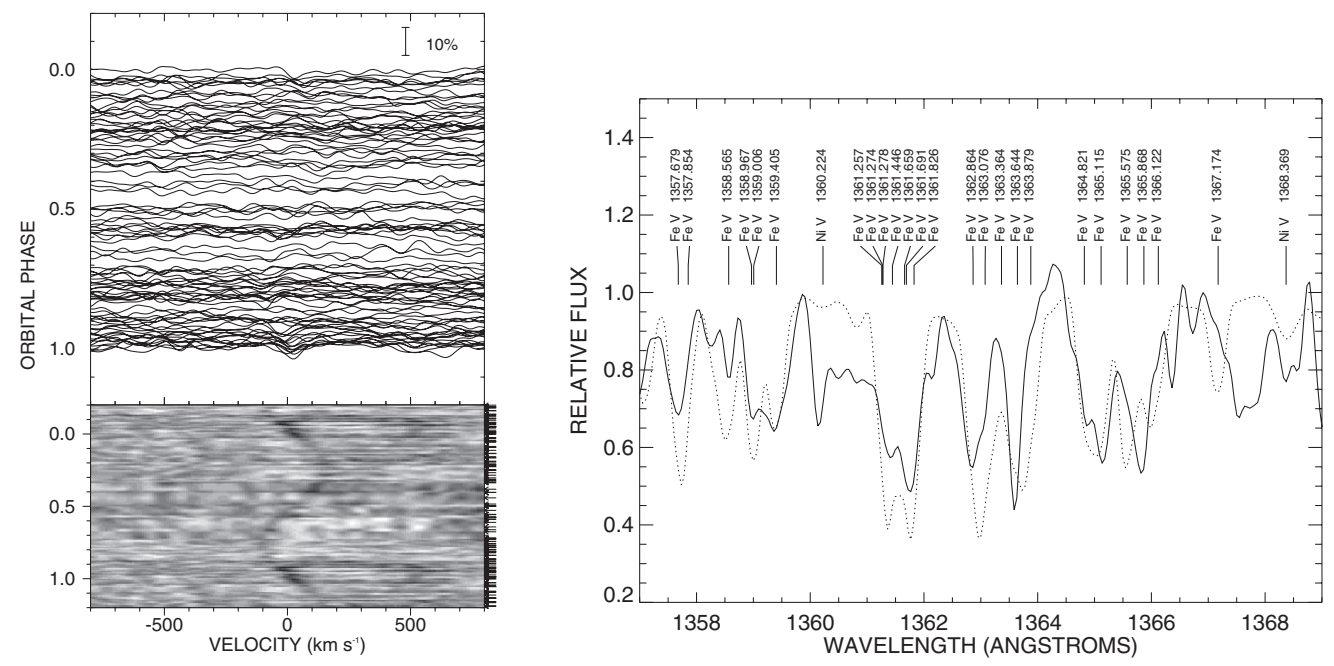

Figure 2. The nature of the subdwarf in the FY CMa system. Left panel: The grayscale at the bottom shows the orbital phase variations in the CCFs in the $1310-1385 \AA$ region produced with a hot star template. The orbital motion of the subdwarf $\left(\mathrm{P}=37^{\mathrm{d}} .25\right)$ is apparent. Right panel: The reconstructed spectrum of the $45 \mathrm{kK}$ subdwarf. From Peters et al. (2008)

of a close hot subdwarf, that would eventually become a massive white dwarf, about a Be star because of the immense difference in their fluxes. But through the analysis of 16 IUE HIRES images of the bright Be star $\phi$ Per (B2 Vpe) with a Doppler tomography code (Bagnuolo et al. 1994), Thaller et al. (1995) were able to recover the spectrum of a hot sdO secondary with a $\mathrm{T}_{\text {eff }}$ of $\sim 50 \mathrm{kK}$. The period of the system is $126 \mathrm{~d} .696$ and the subdwarf contributes only $\sim 15 \%$ of the light in the FUV. We recently employed the same procedures discussed in Thaller et al. (1995) and Gies et al. (1998) to recover the spectrum of a 45,000 K sdO around the B0.5 IVe star FY CMa (Peters et al. 2008). The analysis made use of 97 IUE SWP HIRES images that were obtained from 197995. We cross-correlated each IUE spectrum with a theoretical spectrum computed from TLUSTY/SYNSPEC (Hubeny 1988, Hubeny \& Lanz 1995) to produce the run of crosscorrelation functions (CCFs) shown in Fig. 2. The recovered spectrum of the sdO object is also displayed. In the case of this system the subdwarf contributes only $4 \%$ of the light in the FUV. We are currently in pursuit of other hot subdwarfs that may be hidden in the light of bright Be stars by making use of the extensive IUE archive.

\subsection{Study of Cyclic Long-Term Variability in Algol Systems}

The IUE database is especially valuable for investigating long-term activity in Algol systems. A problem of current interest is finding the cause for the DPV phenomenon that was first identified by Mennickent et al. (2003) from the analysis of OGLE-II observations of Be stars in the LMC. DPV binaries show two photometric periods, one associated with orbital motion and the other a cyclic long-term light modulation that is $\sim 35$ times $\mathrm{P}_{\text {orb }}$. The DPV phenomenon was first observed in the galactic Algols AU Mon (Lorenzi 1980) and RX Cas (Kalv 1979). The 164 SWP/LWP HIRES \& LORES images of AU Mon $\left(\mathrm{B} 3 \mathrm{Ve}+\mathrm{G} \mathrm{IV}, \mathrm{P}_{\text {orb }}=11 . .11\right)$ that exist in the IUE archive are currently being used to shed some light on the DPV phenomenon. Some early results (Peters \& Hageman, in prep.) suggest that the long period light cycle is caused from a waxing/waning of an obscurring accretion disk. In the left panel of Fig. 3 we show a series of IUE LORES spectra taken throughout a primary eclipse when the star was relatively bright in its 

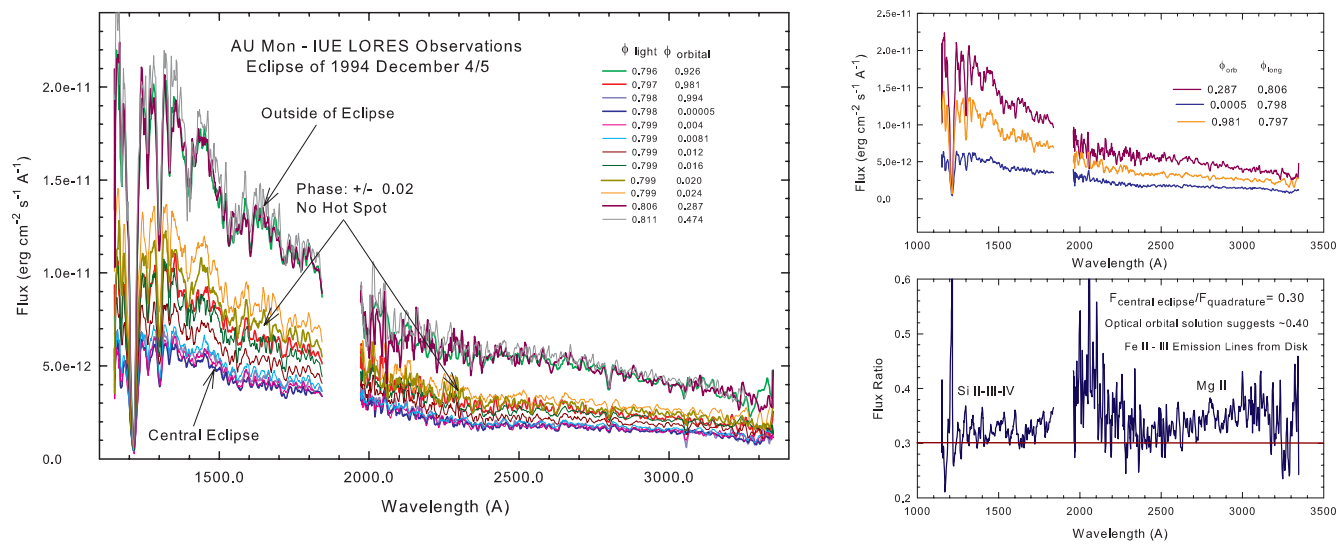

Figure 3. IUE LORES observations of AU Mon taken throughout the 1994 December eclipse (left panel) when the system was relatively bright in its long-term light cycle (maximum brightness is at $\phi_{\text {long }}=0.0$ ) reveal CS emission, symmetry in the eclipse, and the likely presence of significant obscuration of the equatorial region of the B star by the CS disk (right panel).

long-term light cycle. It is immediately apparent that the eclipse was symmetrical (no evidence for a hot accretion spot on the trailing hemisphere) and that the photospheric absorption lines are less visible at primary minimum. The latter suggests that the accretion disk around the B star not only emits strongly in $\mathrm{H} \alpha$ but also in the lines of ionized species in the UV. Such circumstellar (CS) emission features are clearly seen when the spectrum taken at minimum light is divided by a spectrum taken well outside of eclipse (right panel of Fig. 3). The depth of the primary eclipse in the UV implies that $30 \%$ of the B star is uncovered at minimum (the $\mathrm{G}$ star contributes essentially no flux in the UV) in contrast to the $40 \%$ that is suggested by the orbital solution from optical data published by Desmet et al. (2010). This infers the presence of a substantial optically-thick disk, even near light maximum, that obscures the equatorial region. Some other conclusions from our current study are that the optical thickness of the CS disk is greatest at minimum light in the long-term cycle, mass infall is greatest at about $\phi_{\text {long }} \sim 0.25$, mass loss from $\phi_{\text {orb }} \sim 0.5$ is largest when the star is bright, and the photosphere of the B star is substantially deficient in carbon as was implied in the study of the star's high temperature CS plasma (Peters \& Polidan 1984).

Support for this project was provided by NASA Grants NNX10AD60G, NNX07AH56G, and NNX04GC48G, the USC WiSE program, and an international travel grant from the American Astronomical Society.

\section{References}

Bagnuolo, W. G., Jr., et al., 1994, ApJ, 423, 446

Desmet, M., et al., 2010, MNRAS, 401, 418

Gies, D. R., et al., 1998, ApJ, 493, 440

Hubeny, I. 1988, Comput. Phys. Commun. 52, 103

Hubeny, I. \& Lanz, T. 1995, ApJ, 439, 875

Kalv, P. 1979, Tartu Astrof. Obs. Teated, 58, 3

Lorenzi, L. 1980, A\&A, 85, 342

Mennickent, R. E., Pietrzyński, G., Diaz, M., \& Gieren, W. 2003, A\&A, 399, L47

Peters, G. J., Gies, D. R., Grundstrom, E. D., \& McSwain, M. V. 2008, ApJ, 686, 1280

Peters, G. J. \& Polidan, R. S. 1984, ApJ, 283, 745

Thaller, M. L., Bagnuolo, W. G., Jr., Gies, D.. R., \& Penny, L. R. 1995, ApJ, 448, 878 